Article

\title{
Hydrogenation of Phenol over Pt/CNTs: The Effects of Pt Loading and Reaction Solvents
}

\author{
Feng Li ${ }^{1,2}$, Bo Cao ${ }^{1}$, Wenxi Zhu ${ }^{1}$, Hua Song ${ }^{1}$, Keliang Wang ${ }^{2}$ and Cuiqin Li ${ }^{1, *}$ \\ 1 Provincial Key Laboratory of Oil \& Gas Chemical Technology, College of Chemistry \& Chemical \\ Engineering, Northeast Petroleum University, Daqing 163318, China; lifeng@nepu.edu.cn (F.L.); \\ caoboshana@126.com (B.C.); zhuwenxi@stu.nepu.edu.cn (W.Z.); songhua@nepu.edu.cn (H.S.) \\ 2 Key Laboratory of Enhanced Oil \& Gas Recovery of Education Ministry, College of Petroleum Engineering, \\ Northeast Petroleum University, Daqing 163318, China; wangk10608@126.com \\ * Correspondence: licuiqin@nepu.edu.cn; Tel.: +86-459-6504224
}

Academic Editor: Leonarda F. Liotta

Received: 20 March 2017; Accepted: 3 May 2017; Published: 8 May 2017

\begin{abstract}
Carbon nanotubes (CNTs)-supported Pt nanoparticles were prepared with selective deposition of Pt nanoparticles inside and outside CNTs (Pt-in/CNTs and Pt-out/CNTs). The effects of Pt loading and reaction solvents on phenol hydrogenation were investigated. The Pt nanoparticles in Pt-in/CNTs versus Pt-out/CNTs are smaller and better dispersed. The catalytic activity and reuse stability toward phenol hydrogenation both improved markedly. The dichloromethane-water mixture as the reaction solvent, compared with either pure medium, decreased the catalytic activity toward phenol hydrogenation and selectivity of cyclohexanone over Pt-in/CNTs, but significantly improved the catalytic activity toward phenol hydrogenation and selectivity of cyclohexanone over Pt-out/CNTs.
\end{abstract}

Keywords: Pt/CNTs; phenol; hydrogenation; loading method; reaction solvent

\section{Introduction}

Hydrogenation of phenol to cyclohexanone or cyclohexanol is an important chemical reaction in the production of nylon and polyamide resins [1]. It is widely accepted that the hydrogenation of phenol mainly occurs between the phenol chemisorbed on the support and the hydrogen activated on metal nanoparticles. Thus, the support and active particle size play important roles in these supported catalysts for hydrogenation.

To date, varieties of catalysts, including noble metal ( $\mathrm{Pd}$ [2-5], Pt [6], $\mathrm{Ru}$ [7], and $\mathrm{Rh}$ [8]) and non-noble metal (Ni [4,9] and Mo [10]) catalysts, have been developed for this reaction. Among them, noble-metal catalysts are highly active in phenol hydrogenation under mild conditions. The industrially preferred $\mathrm{Pd} / \mathrm{C}$ catalyst has been well studied, including solvents, supports, and various reaction parameters [2-5], but the results of cyclohexanone selectivity are in consistent and even controversial [5]. As is well known, Pt-based catalysts exhibit excellent properties in many hydrogenation reactions. Yang et al. [6] reported the high catalytic activity and cyclohexane selectivity of $\mathrm{Pt} /$ titanate nanotubes in phenol hydrogenation, even at $327 \mathrm{~K}$. However, the application of Pt-based catalysts in phenol hydrogenation is still rarely reported.

Carbon nanotubes (CNTs) has been widely studied as a support of metal catalysts [11-13]. Surface modification of CNTs with oxygenated groups (e.g., carboxylic or hydroxyl) can be simplified by the pretreatment with $\mathrm{HNO}_{3}$, which has been reported as an effective method to optimize the performance of CNT-supported metal catalysts as well as purify and open the tips of CNTs [2]. Matos et al. [14] reported that the phenol hydrogenation produces cyclohexanone over a polar $\mathrm{TiO}_{2}-\mathrm{C}$-supported $\mathrm{Pd}$ catalyst, while it produces cyclohexanol over a hydrophobic $\mathrm{TiO}_{2}-\mathrm{C}$-supported 
catalyst. Makowski et al. [15] reported that the hydrophilic carbon-supported Pd catalyst showed very high cyclohexanone selectivity in phenol hydrogenation. These results suggest that the hydrophilicity/hydrophobicity of CNTs could affect the catalytic properties of CNTs-supported metal catalysts for phenol hydrogenation.

The catalysts with active particles loaded inside and outside CNTs exhibit distinctly different catalytic performances. Research has shown that metal particles (i.e., $\mathrm{Ru}, \mathrm{Co}$, and $\mathrm{Fe}$ ) encapsulated inside CNTs exhibit superior catalytic performance compared with those loaded outside CNTs in hydrogenation [16], oxygen reduction [17], Fischer-Tropsch synthesis [18,19], and polymerization [20]. This superior catalytic performance can be attributed to the unique "confinement effect" of CNTs [21-24].

On the basis of research by Tessonnier et al. [25], here we prepared Pt/CNTs catalysts through selective deposition of $\mathrm{Pt}$ nanoparticles inside and outside CNTs (Pt-in/CNTs and Pt-out/CNTs). For comparison, CNTs-supported $\mathrm{Pt}$ was also prepared via incipient wetness impregnation (Pt-imp/CNTs). Hydrogenation of phenol over different Pt/CNTs catalysts under mild conditions was evaluated to investigate how $\mathrm{Pt}$ loading affected catalytic performances. In addition, by considering the hydrophilicity/hydrophobicity of CNTs, reactants, and products, we studied the effect of a dichloromethane-water mixed solvent on Pt/CNTs catalysts toward phenol hydrogenation.

\section{Results and Discussion}

Figure 1 shows the transmission electron microscopy (TEM) and Pt particle size distribution of the catalysts prepared from different loading methods. Clearly, the Pt particles in Pt-in/CNTs are well dispersed inside CNTs (Figure 1a). The Pt particle distribution is fairly uniform in Pt-in/CNTs and complete on the outer surface of Pt-out/CNTs (Figure 1c), but is random at both inside and outside $\mathrm{Pt}$-imp/CNTs (Figure 1b). The Pt particle sizes in Pt-out/CNTs (10.3 nm) and Pt-imp/CNTs (5.5 nm) are both significantly larger than Pt-in/CNTs $(2.4 \mathrm{~nm})$, indicating that the nano-space in CNTs can effectively inhibit the aggregation of $\mathrm{Pt}$ particles.

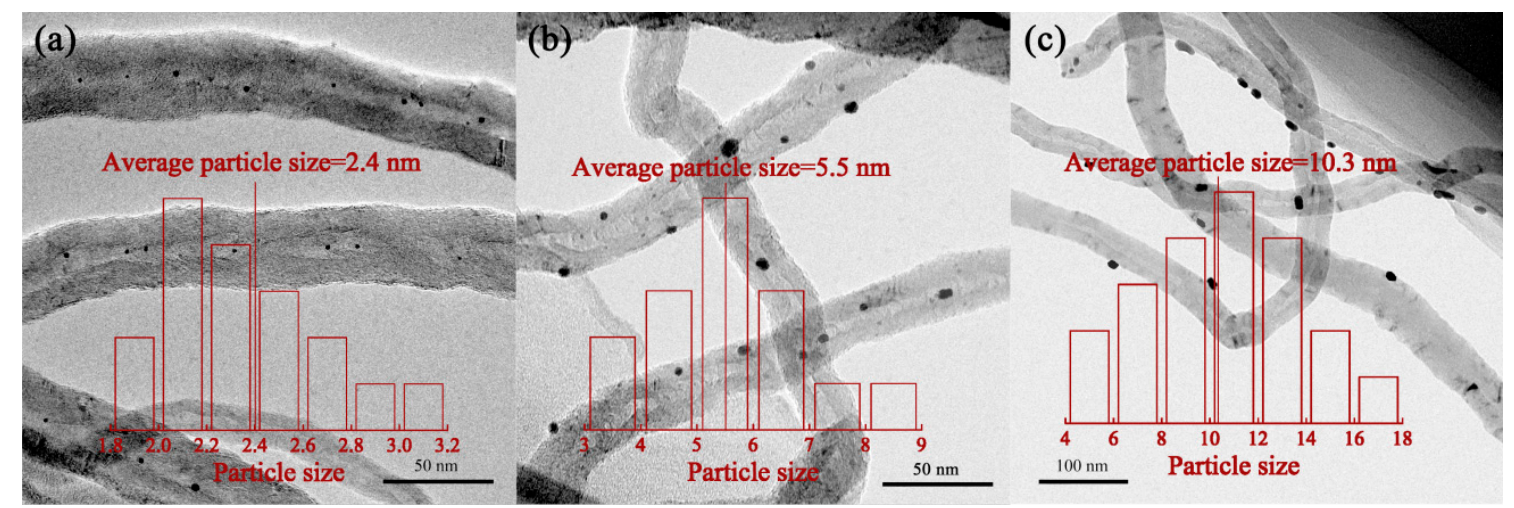

Figure 1. Transmission electron microscopy (TEM) images and particle size distribution of (a) $\mathrm{Pt}$ nanoparticles inside carbon nanotubes (CNTs) (Pt-in/CNTs); (b) Pt nanoparticles prepared via incipient wetness impregnation (Pt-imp/CNTs); (c) Pt nanoparticles outside CNTs (Pt-out/CNTs).

Figure 2 show the $\mathrm{N}_{2}$ adsorption-desorption isotherms and pore size distributions of different catalysts. Clearly, all catalysts have similar Type III isotherms and have an obvious hysteresis loop at relatively high pressures, which is due to the typical capillary condensation caused by the mesopore or accumulated pores in the catalysts. 


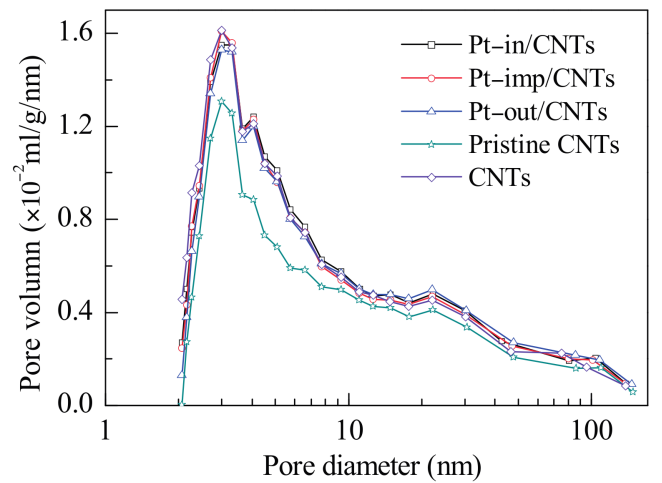

(a)

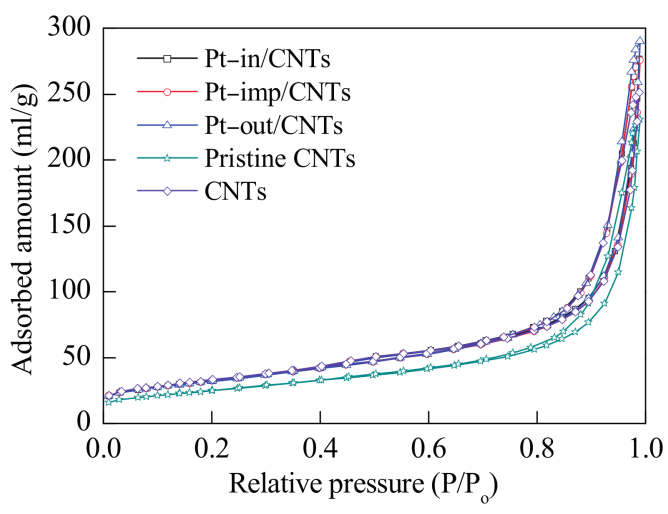

(b)

Figure 2. (a) $\mathrm{N}_{2}$ adsorption-desorption isotherms and (b) pore size distribution curves of $\mathrm{Pt}-\mathrm{in} / \mathrm{CNTs}$, $\mathrm{Pt}$-imp/CNTs, Pt-out/CNTs, pristine CNTs, and CNTs by acid treatment.

Table 1. Physicochemical properties of CNTs and catalysts.

\begin{tabular}{|c|c|c|c|c|c|c|c|}
\hline \multirow{2}{*}{ Sample } & \multirow{2}{*}{$\begin{array}{l}\text { SBET } \\
\left(\mathrm{m}^{2} / \mathrm{g}\right)\end{array}$} & \multirow{2}{*}{$\begin{array}{l}\text { Vpore } \\
\left(\mathrm{cm}^{3} / \mathrm{g}\right)\end{array}$} & \multirow{2}{*}{$\begin{array}{l}\text { Dpore } \\
\text { (nm) }\end{array}$} & \multirow{2}{*}{$\begin{array}{c}\text { Pt } \\
\text { Dispersion }^{1}\end{array}$} & \multicolumn{2}{|c|}{ Average Particle Size of Pt (nm) } & \multirow{2}{*}{$\mathrm{Pt}^{0} / \mathrm{Pt}^{2+, 4}$} \\
\hline & & & & & CO Chemisorption $^{2}$ & TEM $^{3}$ & \\
\hline CNTs $^{5}$ & 87.2 & 0.36 & 16.1 & - & - & - & - \\
\hline CNTs 6 & 114.3 & 0.39 & 13.4 & - & - & - & - \\
\hline Pt-in/CNTs & 112.7 & 0.43 & 14.9 & 0.44 & 2.6 & 2.4 & $52.5 / 47.5$ \\
\hline Pt-imp/CNTs & 112.4 & 0.43 & 14.9 & 0.32 & 3.5 & 5.5 & $35.1 / 64.9$ \\
\hline Pt-out/CNTs & 111.7 & 0.45 & 15.8 & 0.17 & 6.6 & 10.3 & $31.3 / 68.7$ \\
\hline
\end{tabular}

The specific surface areas, pore volumes, and average pore diameters of both CNTs and catalysts are summarized in Table 1. The texture parameters of the CNTs improved after pretreatment with $\mathrm{HNO}_{3}$, which was due to the end opening of the CNTs and the removal of impurities [28]. Compared with acid-treated CNTs, the specific surface areas of the catalysts decreased, but only slightly, because of the low load of $\mathrm{Pt}(3 \mathrm{wt} \%)$. It is noticed that the pore volumes and average pore diameters of the catalysts all increase slightly, which may be due to the decomposition of carbon oxygen compounds during the catalyst synthesis. The Pt dispersion degree in Pt-in/CNTs is higher that that in Pt-out/CNTs and Pt-imp/CNTs.

The X-ray diffraction (XRD) patterns of CNTs and catalysts are shown in Figure 3. The strong diffraction peaks at $26.1^{\circ}$ and $42.9^{\circ}$ are attributed to the hexagonal graphite structure (002) and (100), which suggests CNTs have a hexagonal graphite structure [29]. The Pt-out/CNTs show three diffraction peaks at $39.7^{\circ}, 46.2^{\circ}$ and $67.4^{\circ}$, which are attributed to the $\mathrm{Pt}(111),(200)$, and (220) peaks, respectively, indicating the presence of $\mathrm{Pt}$ in a face-centered cubic (fcc) structure. The Pt (111) crystal plane is more evident in Pt-out/CNTs, and its intensity is obviously stronger than in Pt-in/CNTs and $\mathrm{Pt}$-imp/CNTs. The decrease in peak intensity under the same Pt load indicates that the Pt nanoparticles are small and highly dispersed, which is consistent with TEM spectra.

The reducibility of the catalysts in $\mathrm{H}_{2}$ atmosphere determined by temperature programmed reduction (TPR) experiments is presented in Figure 4. For Pt-in/CNTs, $\mathrm{H}_{2}$ reduction was initiated at $390 \mathrm{~K}$ and the peak appeared at $466 \mathrm{~K}$. This TPR peak can be ascribed to the continual multi-step single-electron reduction of $\mathrm{Pt}^{\mathrm{n}+}$ [30]. The peaks at the temperature above $573 \mathrm{~K}$ can be attributed to the decomposition of the oxygen-containing groups on the surfaces of the CNTs (about 573-973 K) [31] and to the gasification of graphene (about $873 \mathrm{~K}$ ) [32]. The occurrence temperature of reduction peakschange as follows: Pt-out/CNTs $>\mathrm{Pt}$-imp/CNTs $>\mathrm{Pt}$-in/CNTs. The higher temperature of the $\mathrm{Pt}^{4+}$-to- $\mathrm{Pt}^{0}$ reduction indicates the relatively poor dispersion of metal salt precursors. $\mathrm{Pt}^{4+}$ confined 
inside CNTs can be easily reduced because of the confinement $[16,17,33]$ and hydrogen spill-over functional groups [34]. This phenomenon can also be explained by electronic effects. Chen et al. [16] reported that the reduction of $\mathrm{Fe}_{2} \mathrm{O}_{3}$ encapsulated in CNTs were facilitated compared with $\mathrm{Fe}_{2} \mathrm{O}_{3}$ encapsulated out of CNTs and thought the $\pi$ electron density of graphene layers shifted from the inner to the outer surface of CNTs, which resulted in the electron deficiency inside CNTs. Thus, the interaction of $\mathrm{Fe}_{2} \mathrm{O}_{3}$ with the interior CNTs walls is different from that with the exterior walls. This interaction with the encapsulated $\mathrm{Fe}_{2} \mathrm{O}_{3}$, which can destabilize $\mathrm{Fe}_{2} \mathrm{O}_{3}$, can at least partially compensate the electron density loss within the channels.

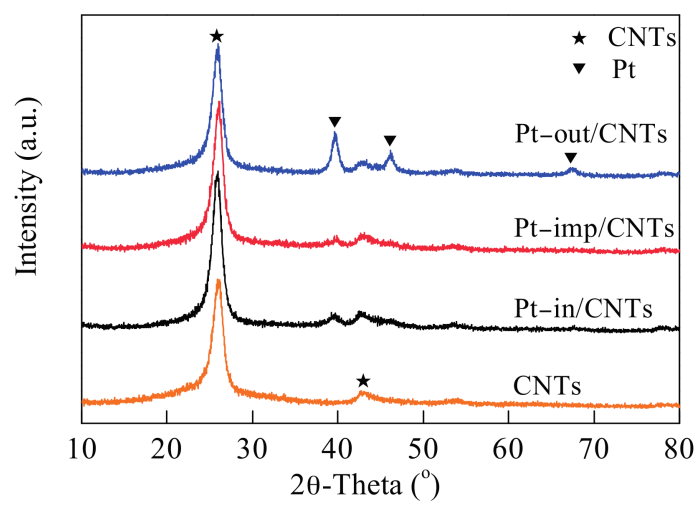

Figure 3. XRD patterns of CNTs, Pt-in/CNTs, Pt-imp/CNTs, and Pt-out/CNTs.

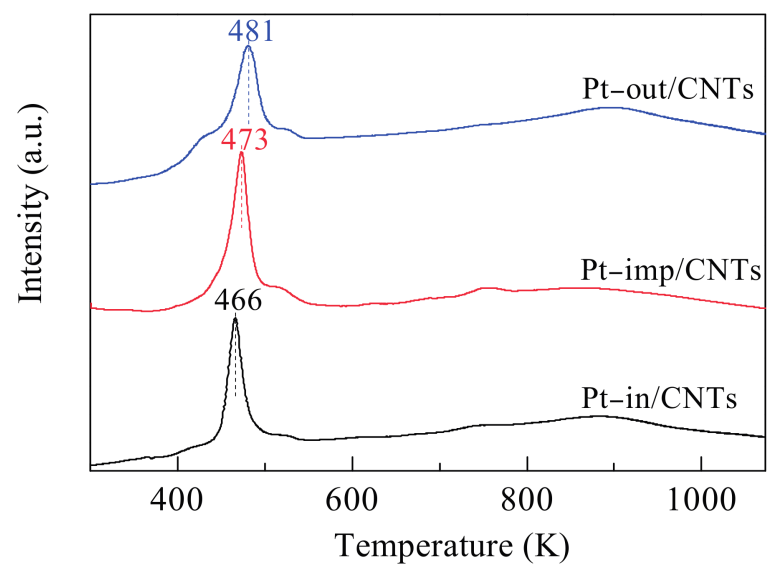

Figure 4. $\mathrm{H}_{2}-\mathrm{TPR}$ (temperature programmed reduction) profiles of $\mathrm{Pt}$-in/CNTs, $\mathrm{Pt}$-imp/CNTs, and Pt-out/CNTs.

As shown in Figure 5, the Pt4f spectra of the catalysts could be deconvoluted into two pairs of doublets attributed to $\mathrm{Pt}^{0}$ and $\mathrm{Pt}^{2+}$, respectively. The binding energy and relative concentration of $\mathrm{Pt}$ species are summarized in Table 2. For Pt-in/CNTs, the doublet at 71.3 and $74.6 \mathrm{eV}$ is attributed to $\mathrm{Pt}^{0}$, and the doublet at 72.0 and $75.7 \mathrm{eV}$ is assigned to $\mathrm{Pt}^{2+}$. By evaluating the compositions of different $\mathrm{Pt}$ species, we find that the percentage of $\mathrm{Pt}^{0} /\left(\mathrm{Pt}^{0}+\mathrm{Pt}^{2+}\right)$ is $52.5 \%$ (Table 1), which indicates the $\mathrm{Pt}$ precursor $\left(\mathrm{H}_{2} \mathrm{PtCl}_{6}\right)$ was reduced mostly to $\mathrm{Pt}^{0}$ and slightly to $\mathrm{Pt}^{2+}$ [35]. In the cases of Pt-imp/CNTs and $\mathrm{Pt}$-out/CNTs, the percentages of $\mathrm{Pt}^{0} /\left(\mathrm{Pt}^{0}+\mathrm{Pt}^{2+}\right)$ are $35.0 \%$ and $31.3 \%$, respectively, indicating that internal loading can promote the reduction of $\mathrm{Pt}^{4+}$ and the formation of $\mathrm{Pt}^{0}$. Chen et al. [16] studied how the confinement in CNTs would affect the activity of Fischer-Tropsch iron catalysts and found that the iron species encapsulated inside CNTs tended to exist in a more reduced state, forming more iron carbides. As for $\mathrm{Pt}$-in/CNTs, the peak of $\mathrm{Pt}^{0}(72.0 \mathrm{eV})$ shifts positively by $0.9 \mathrm{eV}$ compared to pure $\mathrm{Pt}(71.1 \mathrm{eV})$. As for $\mathrm{Pt}$-imp/CNTs and $\mathrm{Pt}$-out/CNTs, the $\mathrm{Pt}^{0}$ peaks for $\mathrm{Pt} 4 \mathrm{f} 7 / 2$ appear at 71.9 and 
$71.7 \mathrm{eV}$, respectively, and the Pt4f7/2 signal shifts positively by 0.8 and $0.6 \mathrm{eV}$ with respect to pure Pt. The positive shift of the $\mathrm{Pt}^{0}$ peak should be attributed to the presence of small nanoparticles [36,37], which agrees well with the TEM spectra in Figure 1.

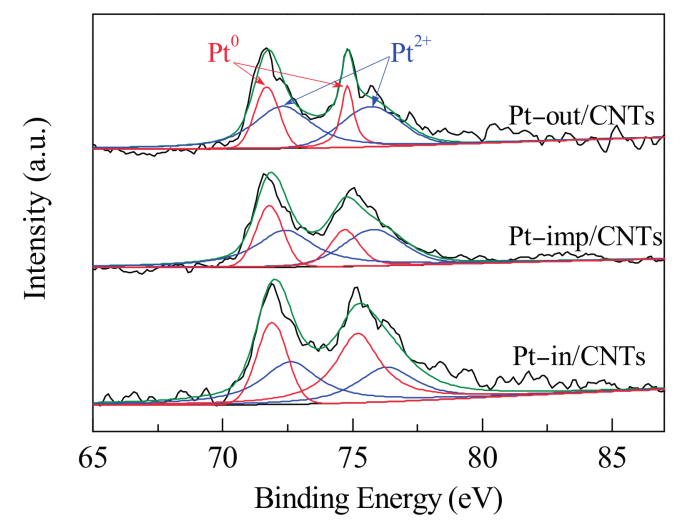

Figure 5. XPS spectra of Pt-in/CNTs, Pt-imp/CNTs, and Pt-out/CNTs.

Table 2 shows the phenol hydrogenation performances of Pt-in/CNTs, Pt-imp/CNTs, and $\mathrm{Pt}$-out/CNTs. Catalytic activity of phenol hydrogenation over the CNTs-supported Pt catalysts ranks as follows: $\mathrm{Pt}$-in/CNTs $>\mathrm{Pt}$-imp/CNTs $>\mathrm{Pt}$-out/CNTs. The activities of phenol hydrogenation are different among the three catalysts with the same Pt load, which can be attributed to the different dispersions. Regarding the structural properties of the CNTs, their nanoscale tubular structure endows them with a unique confinement effect. Given the components with catalytic activity, TEM (Figure 1) and XPS (Figure 5) both show that intratubal loading versus extratubal loading can first effectively inhibit the growth and aggregation of Pt particles, providing larger active specific surfaces under the same $\mathrm{Pt}$ load, and secondly help to increase the concentration of active $\mathrm{Pt}^{0}$ in the catalyst. With regard to catalytic reactions, the phenol hydrogenation occurs inside of $\mathrm{Pt}$-in/CNTs and outside of Pt-out/CNTs. Compared with the outer spaces, the internal nanospaces of CNTs act as a nanoreactor for phenol hydrogenation. Guan et al. [38] reported that the CNTs as a nanoreactor function not only to enrich the molecules inside the channels, but also to stabilize the higher oxidative state of $\mathrm{Pt}$.

Table 2. Hydrogenation of phenol over Pt-in/CNTs, Pt-imp/CNTs, and Pt-out/CNTs ${ }^{1}$.

\begin{tabular}{ccccc}
\hline \multirow{2}{*}{ Catalyst } & \multirow{2}{*}{ Conversion (\%) } & \multicolumn{2}{c}{ Selectivity (\%) } & \multirow{2}{*}{ Reaction Rate $^{\mathbf{2}}$} \\
\cline { 3 - 4 } & & Cyclohexanone & Cyclohexanol & \\
CNTs & $<1$ & - & - & - \\
Pt-in/CNTs & 97.3 & 77.5 & 22.5 & 0.934 \\
Pt-imp/CNTs & 33.8 & 75.3 & 24.7 & 0.324 \\
Pt-out/CNTs & 11.6 & 72.3 & 27.7 & 0.111 \\
\hline
\end{tabular}

${ }^{1}$ Reaction conditions: $20 \mathrm{mg}$ catalyst, $0.5 \mathrm{~g}$ phenol, $0.5 \mathrm{MPa} \mathrm{H}_{2}, 323 \mathrm{~K}, 30 \mathrm{~min} .{ }^{2}$ The phenol mole conversion per mol Pt per second, mol $_{\text {phenol }}$ mol $_{\mathrm{Pt}^{-1}} \mathrm{~s}^{-1}$.

Since the reuse performance of the supported noble metal catalysts is one major factor in practical application, we also examined the catalytic performances of the recycled catalysts (Table 3). After four consecutive cycles, the phenol conversion of Pt-in/CNTs, dropped from 97.3\% to $95.4 \%$, while that of Pt-out/CNTs reduced from $11.6 \%$ to $7.4 \%$, indicating that Pt-in/CNTs had higher reusability than Pt-out/CNTs. On one hand, inductive coupled plasma emission spectra (ICP) analysis (Table 3) showed that the Pt load decreased from $3.02 \mathrm{wt} \%$ to $2.95 \mathrm{wt} \%$ in Pt-in/CNTs, and from $3.01 \mathrm{wt} \%$ to $2.54 \mathrm{wt} \%$ in Pt-out/CNTs. These changes indicate that the loss of Pt particles can be effectively suppressed by intratubal loading more so than extratubal loading. On the other hand, the intratubal space of CNTs can restrict the aggregation of Pt particles. Therefore, the Pt-in/CNTs showed good 
reuse performance. It should also be noted that the selectivity of cyclohexanone increased from $77.5 \%$ to $83.4 \%$ after four consecutive cycles, whereas the conversion was approximately the same.

Table 3. Reusability of catalysts with different loading methods in the hydrogenation of phenol ${ }^{1}$.

\begin{tabular}{lcccccc}
\hline \multirow{2}{*}{ Catalyst } & Run & $\mathbf{P t}$ & Conversion & \multicolumn{2}{c}{ Selectivity (\%) } & \multirow{2}{*}{ Reaction Rate } \\
\cline { 3 - 6 } & & $\mathbf{( w t} \%)$ & $\mathbf{( \% )}$ & CyclohexanoneCyclohexanol & \\
\hline \multirow{3}{*}{ Pt-in/CNTs } & 1 & 3.02 & 97.3 & 77.5 & 22.5 & 0.933 \\
& 3 & - & 96.8 & 80.1 & 19.9 & 0.929 \\
& 4 & 2.95 & 96.0 & 79.9 & 20.1 & 0.921 \\
& 1 & 3.01 & 11.6 & 72.3 & 27.7 & 0.915 \\
\hline \multirow{3}{*}{ Pt-out/CNTs } & 2 & - & 10.5 & 74.5 & 25.5 & 0.111 \\
& 3 & - & 9.0 & 74.6 & 25.4 & 0.101 \\
& 4 & 2.54 & 7.4 & 75.4 & 24.6 & 0.076 \\
\hline
\end{tabular}

${ }^{1}$ Reaction conditions are similar to those listed in Table 2.

After treatment by $\mathrm{HNO}_{3}$, the oxygen-containing groups endowed the CNTs with stronger hydrophilicity, which was unfavorable for catalyst dispersion in dichloromethane. However, in the reaction medium of pure water, the phenol conversion of Pt-imp/CNTs was only $8.8 \%$ (Table 4 ). In pure water, the phenol conversion of both Pt-in/CNTs and Pt-out/CNTs dropped, and the corresponding selectivity of cyclohexanol both increased. The use of the dichloromethane-water solvent efficiently improved the phenol hydrogenation activity of $\mathrm{Pt}$-imp/CNTs. Compared with pure dichloromethane, when the mixed medium contained $10 \mathrm{wt} \%$ water, the phenol conversion of Pt-imp/CNTs increased from $33.8 \%$ to $86.0 \%$, and the corresponding selectivity of cyclohexanone rose from $75.3 \%$ to $99.4 \%$. When the water concentration further rose to $15 \mathrm{wt} \%$, the phenol conversion rose to $89.6 \%$, but the selectivity of cyclohexanone dropped to $94.3 \%$. As for Pt-out/CNTs, when the water concentration was $10 \mathrm{wt} \%$, the phenol conversion increased from $11.6 \%$ to $75.3 \%$, while the selectivity of cyclohexanone rose from $72.3 \%$ to $93.1 \%$. Unlike Pt-imp/CNTs or Pt-out/CNTs, the phenol conversion of Pt-in/CNTs declined from $97.3 \%$ to $72.5 \%$, while the selectivity of cyclohexanone dropped from $77.5 \%$ to $48.9 \%$.

Table 4. Effect of dichloromethane-water mixture on hydrogenation of phenol over Pt-in/CNTs, Pt-imp/CNTs, and Pt-out/CNTs ${ }^{1}$.

\begin{tabular}{|c|c|c|c|c|c|}
\hline \multirow{2}{*}{ Catalyst } & \multirow{2}{*}{$\begin{array}{c}\mathrm{H}_{2} \mathrm{O} \\
(w \mathrm{t} \%)\end{array}$} & \multirow{2}{*}{$\begin{array}{c}\text { Conversion } \\
(\%)\end{array}$} & \multicolumn{2}{|c|}{ Selectivity (\%) } & \multirow{2}{*}{ Reaction Rate } \\
\hline & & & Cyclohexanone & Cyclohexanol & \\
\hline \multirow{8}{*}{ Pt-imp/CNTs } & 5 & 70.6 & 89.5 & 10.5 & 0.677 \\
\hline & 10 & 86.0 & 99.4 & 0.6 & 0.825 \\
\hline & 15 & 89.6 & 94.3 & 5.7 & 0.860 \\
\hline & 20 & 83.7 & 91.2 & 8.8 & 0.803 \\
\hline & 50 & 28.1 & 78.5 & 21.5 & 0.270 \\
\hline & 100 & 8.8 & 2.9 & 97.1 & 0.084 \\
\hline & $100^{2}$ & 16.4 & 17.4 & 82.6 & 0.157 \\
\hline & $100^{3}$ & 35.1 & 42.2 & 57.8 & 0.337 \\
\hline \multirow{2}{*}{$\mathrm{Pt}-\mathrm{in} / \mathrm{CNTs}$} & 10 & 72.5 & 48.9 & 51.1 & 0.696 \\
\hline & 100 & 6.3 & 3.6 & 96.4 & 0.060 \\
\hline \multirow{2}{*}{ Pt-out/CNTs } & 10 & 75.3 & 93.1 & 6.9 & 0.722 \\
\hline & 100 & 8.9 & 7.6 & 93.4 & 0.085 \\
\hline
\end{tabular}

${ }^{1}$ Reaction conditions: $10 \mathrm{~mL}$ of dichloromethane-water mixture, other reaction conditions are similar to those listed in Table 2. ${ }^{2,3}$ Reaction temperatures are 333 and $343 \mathrm{~K}$, respectively.

The mechanism of phenol hydrogenation over Pt-in/CNTs and Pt-out/CNTs are shown in Figure 6. Under high-speed stirring, the dichloromethane-water mixed system reached a latex phase, 
where the water phase was dispersed in the form of nuclei, while the hydrophilicity of CNTs improved the catalyst dispersity in water. Upon the dichloromethane/water interfaces, the interaction between the hydroxyl groups of phenol and the hydroxyl groups of CNTs enhanced the phenol absorbability onto the catalyst. Under the action of extratubally loaded $\mathrm{Pt}$, the cyclohexanone resulting from hydrogenation became less water-soluble, but dissolved more in the dichloromethane. Thus, under the stirring condition, the cyclohexanone migrated to the dichloromethane, which reduced further hydrogenation and improved the cyclohexanone selectivity. For the intratubally loaded $\mathrm{Pt}$, however, the hydrophilicity of CNTs and the low water solubility of phenol (reaction at $323 \mathrm{~K}$ ) inhibited the contact between active component and phenol. Moreover, the cyclohexanone resulting from phenol hydrogenation could not spread to the dichloromethane in time, but instead was adsorbed intratubally into the nanoscale structures of CNTs, further promoting the hydrogenation to form cyclohexanol. Immediately after its formation, hydroxyl groups of cyclohexanol further reacted with the hydroxyl groups of CNTs to form hydrogen bonds, which led to a competitive catalyst surface adsorption between cyclohexanol and phenol. As the reactions proceeded, the amount of cyclohexanol formed in the catalyst increased, and the amount of adsorbed phenol declined, leading to a decline of phenol conversion and an increase in cyclohexanol selectivity. These changes also account for the low phenol conversion and the high cyclohexanol selectivity in pure water.

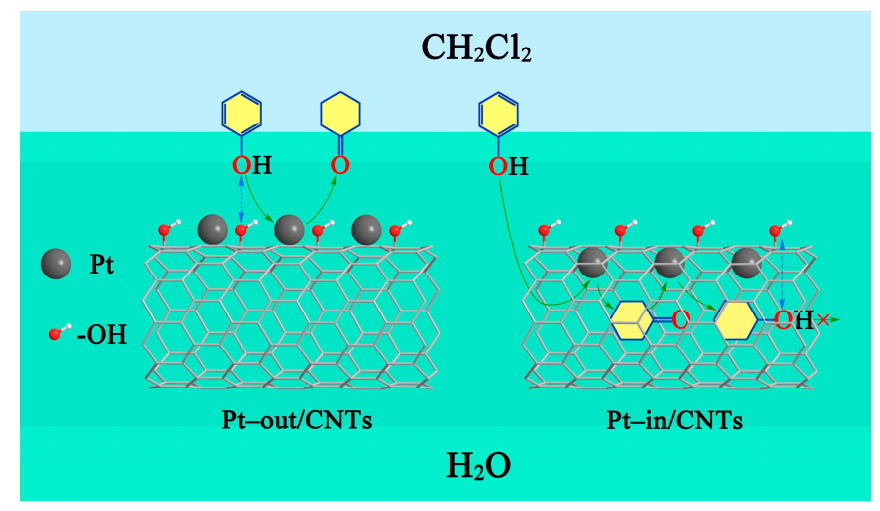

Figure 6. Mechanism of phenol hydrogenation over Pt-in/CNTs and Pt-out/CNTs.

\section{Experimental}

\subsection{Chemicals}

CNTs were purchased from Shenzhen Nanotech Port Co., Ltd. (Shenzhen, China) $\mathrm{H}_{2} \mathrm{PtCl}_{6}$ were purchased from Shenyang Jinke Reagent Company (Shenyang, China) and $\mathrm{CH}_{2} \mathrm{Cl}_{2}$ from Shenyang Huadong Reagent Company (Shenyang, China). Ethanol, phenol and $\mathrm{HNO}_{3}$ were purchased from Haerbin chemical Reagent Company (Harbin, China). All the chemicals were analytical grade with no further treatment. Deionized water was used for solution preparation.

\subsection{Catalyst Preparation}

Based on the difference in the interface energies of ethanol and water with the CNTs surface, $\mathrm{Pt}$ particles were selectively deposited inside and outside CNTs, which were denoted as Pt-in/CNTs and Pt-out/CNTs. Prior to impregnation, pristine CNTs were treated with $\mathrm{HNO}_{3}$ reflux at $398 \mathrm{~K}$ for $6 \mathrm{~h}$. ForPt-in/CNTs, $0.1 \mathrm{~g}$ of CNTs were impregnated with $0.35 \mathrm{~mL}$ of $\mathrm{H}_{2} \mathrm{PtCl}_{6}$ ethanol solution (about 2/3 of CNTs saturated water absorption rate $(5.23 \mathrm{~mL} / \mathrm{g}))$ at $293 \mathrm{~K}$, followed by adding of $0.26 \mathrm{~mL}$ of $\mathrm{H}_{2} \mathrm{O}$. After being dried, the samples were treated in $\mathrm{H}_{2}$ at $573 \mathrm{~K}$ for $3 \mathrm{~h}$ to reduce the Pt precursors into $\mathrm{Pt}$ metal. For Pt-out/CNTs, $0.1 \mathrm{~g}$ of CNTs were impregnated with $0.52 \mathrm{~mL}$ of ethanol, followed by an addition of $0.35 \mathrm{~mL}$ of $\mathrm{H}_{2} \mathrm{PtCl}_{6}$ aqueous solution. The drying and reducing process were performed the same as for Pt-in/CNTs. For comparison, CNTs-supported Pt was also prepared by incipient 
wetness impregnation with an $\mathrm{H}_{2} \mathrm{PtCl}_{6}$ aqueous solution, which was denoted as $\mathrm{Pt}$-imp/CNTs. The Pt theoretical load was $3 \mathrm{wt} \%$.

\subsection{Catalyst Characterization}

TEM was performed on a JEOL JEM-4000EX microscope (JEOL, Tokyo, Japan). $\mathrm{N}_{2}$ physisorption measurements were carried out using a TristarII 3020 surface area and porosity analyzer (Micromeritic, Atlanta, GA, USA). Pore size distributions were obtained from the isotherm adsorption branch, using the Barrett-Joyner-Halenda model. The Pt dispersion, expressed in terms of CO chemisorbed/Pttotal, was calculated by assuming a CO-to-surface Pt atom ratio of 1:1 [26]. XRD was performed on a Rigaku $\mathrm{D} / \mathrm{max}-2200 \mathrm{PC}$ diffractometer (Rigaku, Tokyo, Japan) using CuK $\alpha$ radiation. TPR was performed on a Chem BET 3000 chemical adsorption instrument (Quantachrome, Boynton Beach, FL, USA) by heating the sample from ambient temperature to $1073 \mathrm{~K}$ at $10 \mathrm{~K} / \mathrm{min}$ in a stream of $5 \mathrm{vol} \% \mathrm{H}_{2} / \mathrm{N}_{2}$ mixture $(40 \mathrm{~mL} / \mathrm{min})$. XPS spectra were attained with a Thermofisher Scientific K-Alpha instrument (Thermofisher, New York, NY, USA). The Pt load (wt \%) was analyzed by means of an ICPS-7510 inductively coupled plasma spectrometer (Shimadzu, Tokyo, Japan).

\subsection{Catalytic Hydrogenation Activity Test}

Hydrogenation of phenol was carried out in a $50 \mathrm{~mL}$ stainless steel autoclave with a Teflon inner layer. Twenty milli grams of catalyst was dispersed in a $10 \mathrm{~mL}$ dichloromethane (or dichloromethane-water mixture) solution of $0.5 \mathrm{~g}$ of phenol. The reactor was sealed and purged with $\mathrm{H}_{2}$ six times. Then, the reaction was carried out at $323 \mathrm{~K}$ for 30 min with $0.5 \mathrm{MPa}_{2}$ at a stirring speed of $700 \mathrm{rpm}$. After cooling to ambient temperature, the liquid reactants were analyzed by gas chromatogram (GC-14, Shimadzu, Tokyo, Japan) with a $30 \mathrm{~m}$ capillary column (DB-WAX) using a flame ionization detector, and were identified by gas chromatography/mass spectrometry (GC/MS, Agilent 5890, Santa Clara, CA, USA).

\section{Conclusions}

$\mathrm{Pt} / \mathrm{CNTs}$ catalysts, with selective deposition of Pt nanoparticles inside and outside CNTs, were prepared based on the difference in the interface energies of ethanol and water with the CNTs surface. Intratubal loading, compared to extratubal loading, features a smaller Pt particle size, a greater dispersity, greater phenol hydrogenation activity, greater cyclohexanone selectivity, and more effectively inhibited the loss of active components and increased catalyst stability. For Pt/CNTs prepared by extratubal loading, using the dichloromethane-water mixture as the reaction solvent significantly improved the catalytic activity for phenol hydrogenation and selectivity of cyclohexanone. The phenol hydrogenation activity and cyclohexanone selectivity of the Pt/CNTs prepared from incipient wetness impregnation were significantly improved by the dichloromethane-water mixture containing $10 \mathrm{wt} \%$ water.

Acknowledgments: The authors acknowledge the financial supports from the China Petroleum and Chemical Industry Federation (2016-09-01).

Author Contributions: Feng Li, Cuiqin Li, and Hua Song conceived and designed the experiments; Feng Li, Bo Cao, and Wenxi Zhu performed the experiments; Keliang Wang and Cuiqin Li analyzed the data; Feng Li contributed reagents/materials/analysis tools; Feng Li and Cuiqin Li wrote the paper.

Conflicts of Interest: The authors declare no conflict of interest.

\section{References}

1. Nie, R.; Miao, M.; Du, W.; Shi, J.; Liu, Y.; Hou, Z. Selective hydrogenation of C=C bond over N-doped reduced graphene oxides supported Pd catalyst. Appl. Catal. B Environ. 2016, 180, 607-613. [CrossRef]

2. Zhang, L.; Wang, B.; Ding, Y.; Wen, G.; Hamid, S.B.A.; Su, D. Disintefrative activation of Pd nanoparticles on carbon nanotubes for catalytic phenol hydrogenation. Catal. Sci. Technol. 2016, 6, 1003-1006. [CrossRef] 
3. Chen, J.; Zhang, W.; Chen, L.; Ma, L.; Gao, H.; Wang, T. Direct selective hydrogenation of phenol and derivatives over polyaniline-functionalized carbon-nanotube-supported palladium. ChemPlusChem 2013, 78, 142-148. [CrossRef]

4. Xiang, Y.; Kong, L.; Xie, P.; Xu, T.; Wang, J.; Li, X. Carbon nanotubes and activated carbons supported catalysts for phenol in situ hydrogenation: Hydrophobic/hydrophilic effect. Ind. Eng. Chem. Res. 2014, 53, 2197-2203. [CrossRef]

5. Xu, T.; Zhang, Q.; Cen, J.; Xiang, Y.; Li, X. Selectivity tailoring of Pd/CNTs in phenol hydrogenation by surface modification: Role of C-O oxygen species. Appl. Surf. Sci. 2015, 324, 634-639. [CrossRef]

6. Yang, X.; Yu, X.; Long, L.; Wang, T.; Ma, L.; Wu, L.; Bai, Y.; Li, X.; Liao, S. Pt nanoparticles entrapped in titanate nanotubes (TNT) for phenol hydrogenation: The confinement effect of TNT. Chem. Commun. 2014, 50, 2794-2796. [CrossRef] [PubMed]

7. Ertas, I.E.; Gulcan, M.; Bulut, A.; Yurderi, M.; Zahmakiran, M. Metal-organic framework (MIL-101) stabilized ruthenium nanoparticles: Highly efficient catalytic material in the phenol hydrogenation. Microporous Mesoporous Mater. 2016, 226, 94-103. [CrossRef]

8. Kuklin, S.; Maximov, A.; Zolotukhina, A.; Karakhanov, E. New approach for highly selective hydrogenation of phenol to cyclohexanone: Combination of rhodium nanoparticles and cyclodextrins. Catal. Commun. 2016, 73, 63-68. [CrossRef]

9. Zhang, Q.; Li, H.; Gao, P.; Wang, L. PVP-NiB amorphous catalyst for selective hydrogenation of phenol and its derivatives. Chin. J. Catal. 2014, 35, 1793-1799. [CrossRef]

10. Boullosa-Eiras, S.; Lødeng, R.; Bergem, H.; Stöcker, M.; Hannevold, L.; Blekkan, E.A. Catalytic hydrodeoxygenation (HDO) of phenol over supported molybdenum carbide, nitride, phosphide and oxide catalysts. Catal. Today 2014, 223, 44-53. [CrossRef]

11. Melchionna, M.; Marchesan, S.; Prato, M.; Fornasiero, P. Carbon nanotubes and catalysis: The many facets of a successful marriage. Catal. Sci. Technol. 2015, 46, 3859-3875. [CrossRef]

12. Wildgoose, G.G.; Banks, C.E.; Compton, R.G. Metal nanoparticles and related materials supported on carbon nanotubes: Methods and applications. Small 2006, 2, 182-193. [CrossRef] [PubMed]

13. Melchionna, M.; Prato, M. Functionalizing carbon nanotubes: An indispensible step towards applications. ECSJ. Solid State Sci. Technol. 2013, 2, M3040-M3045. [CrossRef]

14. Matos, J.; Corma, A. Selective phenol hydrogenation in aqueous phase on Pd-based catalysts supported on hybrid $\mathrm{TiO}_{2}$-carbon materials. Appl. Catal. A Gen. 2011, 404, 103-112. [CrossRef]

15. Makowski, P.; Cakan, R.D.; Antonietti, M.; Goettmann, F.; Titirici, M.M. Selective partial hydrogenation of hydroxy aromatic derivatives with palladium nanoparticles supported on hydrophilic carbon. Chem. Commun. 2008, 8, 999-1001. [CrossRef] [PubMed]

16. Li, F.; Ma, R.; Cao, B.; Liang, J.; Ren, Q.; Song, H. Effect of Co-B supporting methods on the hydrogenation of m-chloronitrobenzene over Co-B/CNTs amorphous alloy catalysts. Appl. Catal. A Gen. 2016, 514, 248-252. [CrossRef]

17. Deng, D.; Yu, L.; Chen, X.; Wang, G.; Jin, L.; Pan, X.; Deng, J.; Sun, G.; Bao, X. Iron encapsulated within pod-like carbon nanotubes for oxygen reduction reaction. Angew. Chem. Int. Ed. 2013, 52, 371-375. [CrossRef] [PubMed]

18. Chen, W.; Fan, Z.; Pan, X.; Bao, X. Effect of confinement in carbon nanotubes on the activity of Fischer-Tropsch iron Catalyst. J. Am. Chem. Soc. 2008, 130, 9414-9419. [CrossRef] [PubMed]

19. Tavasoli, A.; Trépanier, M.; Dalai, A.K.; Abatzoglou, N. Effect of confinement in carbon nanotubes on the activity, selectivity, and lifetime of Fischer-Tropsch Co/carbon nanotube catalysts. J. Chem. Eng. Data 2010, 55, 2757-2763. [CrossRef]

20. Li, X.; Zhang, L.; Tan, R.P.; Fazzini, P.F.; Hungria, T.; Durand, J.; Lachaize, S.; Sun, W.H.; Respaud, M.; Soulantica, K.; Serp, P. Isoprene polymerization on iron nanoparticles confined in carbon nanotubes. Chem. Eur. J. 2015, 21, 17437-17444. [CrossRef] [PubMed]

21. Castillejos, E.; Debouttière, P.J.; Roiban, L.; Solhy, A.; Martinez, V.; Kihn, Y.; Ersen, O.; Philippot, K.; Chaudret, B.; Serp, P. An efficient strategy to drive nanoparticles into carbon nanotubes and the remarkable effect of confinement on their catalytic performance. Angew. Chem. Int. Ed. 2009, 48, 2529-2533. [CrossRef] [PubMed]

22. Ha, K.S.; Kwak, G.; Jun, K.W.; Hwang, J.; Lee, J. Ordered mesoporous carbon nanochannel reactors for high-performance Fischer-Tropsch synthesis. Chem. Commun. 2013, 49, 5141-5143. [CrossRef] [PubMed] 
23. Lemus-Yegres, L.J.; Román-Martínez, M.C.; Such-Basáñez, I.; Lecea, C.S.M. Effects of confinement in hybrid diamine-Rh complex-carbon catalysts used for hydrogenation reactions. Microporous Mesoporous Mater. 2008, 109, 305-316. [CrossRef]

24. Xiao, J.; Pan, X.; Guo, S.; Ren, P.; Bao, X. Toward fundamentals of confined catalysis in carbon nanotubes. J. Am. Chem. Soc. 2015, 137, 477-482. [CrossRef] [PubMed]

25. Tessonnier, J.P.; Ersen, O.; Weinberg, G.; Pham-Huu, C.; Su, D.S.; Schlögl, R. Selective deposition of metal nanoparticles inside or outside multiwalled carbon nanotubes. ACS Nano 2009, 3, 2081-2089. [CrossRef] [PubMed]

26. Haneda, M.; Watanabe, T.; Kamiuchi, N.; Ozawa, M. Effect of platinum dispersion on the catalytic activity of $\mathrm{Pt} / \mathrm{Al}_{2} \mathrm{O}_{3}$ for the oxidation of carbon monoxide and propene. Appl. Catal. B Environ. 2013, 142-143, 8-14. [CrossRef]

27. Hu, C.; Creaser, D.; Fouladvand, S.; Grönbeck, H.; Skoglundh, M. Methyl crotonate hydrogenation over Pt: Effects of support and metal dispersion. Appl. Catal. A Gen. 2016, 511, 106-116. [CrossRef]

28. Shi, L.; Chu, W.; Deng, S. Catalytic properties of Cu-Co catalysts supported on $\mathrm{HNO}_{3}$-pretreated CNTs for higher-alcohol synthesis. J. Nat. Gas Chem. 2011, 20, 48-52. [CrossRef]

29. Arciniega Cano, O.; Rodríguez González, C.A.; Hernández Paz, J.F.; Amezaga Madrid, P.; García Casillas, P.E.; Martínez Hernández, A.L.; Martínez Pérez, C.A. Catalytic activity of palladium nanocubes/multiwalled carbon nanotubes structures for methyl orange dye removal. Catal. Today 2017, 282, 168-173. [CrossRef]

30. Yang, L.; Lin, G.D.; Zhang, H.B. Highly efficient Pd-ZnO catalyst doubly promoted by CNTs and $\mathrm{Sc}_{2} \mathrm{O}_{3}$ for methanol steam reforming. Appl. Catal. A Gen. 2013, 455, 137-144. [CrossRef]

31. Li, C.; Shao, Z.; Pang, M.; Williams, C.T.; Liang, C. Carbon nanotubes supported Pt catalysts for phenylacetylene hydrogenation: Effects of oxygen containing surface groups on Pt dispersion and catalytic performance. Catal. Today 2012, 186, 69-75. [CrossRef]

32. Qu, P.F.; Chen, J.G.; Song, Y.H.; Liu, Z.T.; Liu, Z.W.; Li, Y.; Lu, J.; Jiang, J. Effect of Fe(III) on hydrogenation of citral over Pt supported multiwalled carbon nanotube. Catal. Commun. 2015, 68, 105-109. [CrossRef]

33. Zhang, Y.; Liu, Y.; Yang, G.; Endo, Y.; Tsubaki, N. The solvent effects during preparation of Fischer-Tropsch synthesis catalysts: Improvement of reducibility, dispersion of supported cobalt and stability of catalyst. Catal. Today 2009, 142, 85-89. [CrossRef]

34. Davari, M.; Karimi, S.; Tavasoli, A.; Karimi, A. CNTs-supported cobalt catalyst in Fischer-Tropsch via CNTs functionalization. Appl. Catal. A Gen. 2014, 485, 133-142. [CrossRef]

35. Peng, R.; Sun, X.; Li, S.; Chen, L.; Fu, M.; Wu, J.; Ye, D. Shape effect of $\mathrm{Pt} / \mathrm{CeO}_{2}$ catalysts on the catalytic oxidation of toluene. Chem. Eng. J. 2016, 306, 1234-1246. [CrossRef]

36. Zhang, C.; Wang, T.; Liu, X.; Ding, Y. Cu-promoted Pt/activated carbon catalyst for glycerol oxidation to lactic acid. J. Mol. Catal. A Chem. 2016, 424, 91-97. [CrossRef]

37. Ryabenkoca, Y.; He, Q.; Miedziak, P.J.; Dummer, N.F.; Taylor, S.H.; Carley, A.F.; Morgan, D.J.; Dimitratos, N.; Willock, D.J.; Bethell, D. The selective oxidation of 1,2-propanediol to lactic acid using mild conditions and gold-based nanoparticulate catalysts. Catal. Today 2013, 203, 139-145. [CrossRef]

38. Guan, Z.; Lu, S.; Li, C. Highly oxidized Pt species stabilized inside carbon nanotubes for asymmetric hydrogenation. Chin. J. Catal. 2015, 36, 1535-1542. [CrossRef]

(C) 2017 by the authors. Licensee MDPI, Basel, Switzerland. This article is an open access article distributed under the terms and conditions of the Creative Commons Attribution (CC BY) license (http://creativecommons.org/licenses/by/4.0/). 